\title{
Comprehensive Analysis of TIFY Transcription Factors and Their Expression Profiles under Jasmonic Acid and Abiotic Stresses in Watermelon
}

\author{
Youxin Yang, ${ }^{1}$ Golam Jalal Ahammed $\mathbb{D}^{2}{ }^{2}$ Chunpeng Wan $\left(\mathbb{D},{ }^{1}\right.$ Haoju Liu, ${ }^{3}$ \\ Rongrong Chen $\left(\mathbb{D},{ }^{3}\right.$ and Yong Zhou $\mathbb{C}^{3,4}$ \\ ${ }^{1}$ Jiangxi Key Laboratory for Postharvest Technology and Nondestructive Testing of Fruits \& Vegetables, Collaborative Innovation \\ Center of Postharvest Key Technology and Quality Safety of Fruits and Vegetables, College of Agronomy, \\ Jiangxi Agricultural University, Nanchang 330045, China \\ ${ }^{2}$ College of Forestry, Henan University of Science and Technology, Luoyang 471023, China \\ ${ }^{3}$ College of Bioscience and Bioengineering, Jiangxi Agricultural University, Nanchang 330045, China \\ ${ }^{4}$ Key Laboratory of Crop Physiology, Ecology, and Genetic Breeding, Ministry of Education, Jiangxi Agricultural University, \\ Nanchang 330045, China \\ Correspondence should be addressed to Rongrong Chen; r_r_chen@163.com and Yong Zhou; yzhoujxau@163.com
}

Received 18 April 2019; Accepted 26 August 2019; Published 1 October 2019

Academic Editor: Wilfred van IJcken

Copyright (C) 2019 Youxin Yang et al. This is an open access article distributed under the Creative Commons Attribution License, which permits unrestricted use, distribution, and reproduction in any medium, provided the original work is properly cited.

The TIFY gene family is plant-specific and encodes proteins involved in the regulation of multiple biological processes. Here, we identified 15 TIFY genes in the watermelon genome, which were divided into four subfamilies (eight JAZs, four ZMLs, two TIFYs, and one PPD) in the phylogenetic tree. The ClTIFY genes were unevenly located on eight chromosomes, and three segmental duplication events and one tandem duplication event were identified, suggesting that gene duplication plays a vital role in the expansion of the TIFY gene family in watermelon. Further analysis of the protein architectures, conserved domains, and gene structures provided additional clues for understanding the putative functions of the TIFY family members. Analysis of qRT-PCR and RNA-seq data revealed that the detected ClTIFY genes had preferential expression in specific tissues. qRT-PCR analysis revealed that nine selected TIFY genes were responsive to jasmonic acid (JA) and abiotic stresses including salt and drought. JA activated eight genes and suppressed one gene, among which ClJAZ1 and ClJAZ7 were the most significantly induced. Salt and drought stress activated nearly all the detected genes to different degrees. These results lay a foundation for further functional characterization of TIFY family genes in Citrullus lanatus.

\section{Introduction}

TIFY transcription factors are plant-specific transcriptional regulators characterized by the presence of a highly conserved motif (TIF(F/Y)XG) in the TIFY domain (previously called ZIM domain) with a length of approximately 36 amino acids (aa) $[1,2]$. According to their domain architectures, the TIFY proteins can be divided into four subfamilies, including TIFY, ZIM-like (ZML), jasmonate ZIM-domain (JAZ), and PEAPOD (PPD). Except for the TIFY subfamily, which only harbors the TIFY domain, members of other three subfamilies possess some additional domains [3]. For example, the
ZML subfamily proteins possess a GATA zinc-finger DNAbinding domain and a CCT domain (CONSTANS, CO-like, and TOC1) $[4,5]$. The JAZ subfamily proteins have a conserved jasmonic acid- (JA-) associated domain (Jas, also known as CCT-2 motif) with a $\mathrm{SLX}_{2} \mathrm{FX}_{2} \mathrm{KRX}_{2} \mathrm{RX}_{5} \mathrm{PY}$ (X represents any amino acid) consensus sequence, while the PPD subfamily proteins contain a typical PPD domain in the $\mathrm{N}$-terminus and a truncated Jas motif lacking the conserved $\mathrm{P}$ and $\mathrm{Y}$ residues [6-8].

To date, a number of TIFY genes have been functionally characterized in Arabidopsis [9-12], rice [13-15], wheat $[16,17]$, tomato [18], cotton [19], and other plants [20-22]. 
AtTIFY1/AtZIM is the first identified TIFY gene in plants, and overexpression of AtTIFY1/AtZIM promotes the elongation of the petiole and hypocotyl, which is independent of gibberellin and brassinosteroids [4, 23]. AtZML1/AtTIFY2b and AtZML2/AtTIFY2a are important components of the CRYPTOCHROME1- (cry1-) mediated response to excess light [24]. In tomato, SIJAZ2 was described as an important regulator of the transition from vegetative growth to reproductive growth [18]. In addition, many $J A Z$ genes were found to play key roles in JA signal transduction and participate in the regulation of various developmental processes and responses to biotic and abiotic stresses in plants. For example, OsTIFY3/OsJAZ1 can regulate floral development and root elongation by interacting with OsMYC2 and OsCOI1b in rice, and substitution or deletion of the core segments of OsJAZ1 can affect the specificity and sensitivity of JA signaling during flower and root development $[13,25]$. In addition, OsJAZ1 was identified as a negative regulator of rice drought resistance, partially by regulating the JA and ABA signaling pathways [14]. Another rice JAZ member, OsJAZ8, can confer resistance to rice bacterial blight by regulating JAresponsive volatile compounds [26]. Additionally, rice plants expressing OsJAZ8 and OsJAZ8 $\Delta \mathrm{C}$ (C-terminal truncated) under the control of the salt-inducible ZOS3-11 promoter exhibited higher tolerance to salt stress in early stages [27].

Recently, genome-wide surveys of the TIFY gene family have been conducted in various plant species such as Brachypodium distachyon [28], maize [29], moso bamboo (Phyllostachys edulis) [30], pigeon pea (Cajanus cajan) [31], Brassica rapa [5], tomato (Solanum lycopersicum) [32], Gossypium species [33], wheat (Triticum aestivum) [16], poplar (Populus trichocarpa) [6, 34], and pear (Pyrus pyrifolia) [35]. However, no genome-wide information of this gene family is available in watermelon, an important agricultural crop susceptible to various biotic and abiotic stresses during growth and development [36].

In this study, we predicted and classified the TIFY family genes of watermelon and analyzed their distribution patterns, phylogenetic relationships, and protein and gene structures. In addition, we examined the expression profiles of several CITIFY genes in various tissues during fruit development of watermelon as well as under diverse abiotic stresses. Our findings lay a solid foundation for further understanding the role of TIFY genes in the growth and development of watermelon.

\section{Materials and Methods}

2.1. Genome-Wide Identification of TIFY Genes in Watermelon. To identify the TIFY family members in watermelon, the hidden Markov model (HMM) profiles of the TIFY domain (PF06200), Jas domain (PF09425), and CCT domain (PF06203) were obtained from the Pfam database (http://pfam.sanger.ac.uk/), and these domains were used as queries to search the watermelon genome database (http:// cucurbitgenomics.org/organism/1) using HMMER 3.0 software (http://hmmer.org/) with an $E$ value cutoff of $1 e^{-5}$. Arabidopsis and rice TIFY protein sequences were also used as queries to obtain the watermelon TIFYs by BlastP through searching the watermelon genome database with a cutoff $E$ value of $1 e^{-5}$. According to previous reports [2, 37], Arabidopsis and rice TIFY protein sequences were downloaded from the Arabidopsis genome database at TAIR (The Arabidopsis Information Resource, https://www .arabidopsis.org/) and from the rice genome database at RGAP (Rice Genome Annotation Project, http://rice .plantbiology.msu.edu/), respectively. The nonredundant sequences were subsequently confirmed with the Pfam database and the SMART database (http://smart.emblheidelberg.de/).

2.2. Protein Properties, Sequence Analyses, and Phylogenetic Tree Construction. The biochemical features including isoelectric point (pI) and molecular weight (MW) of watermelon TIFY proteins were calculated with the ProtParam tool (http://web.expasy.org/protparam/). Conserved motifs were identified using the MEME tool (http://meme-suite .org/tools/meme) with the maximum number of motifs set as 10. The exon-intron structures of watermelon TIFY genes were displayed by the GSDS tool (http://gsds.cbi.pku.edu.cn) based on the alignment of coding region sequences (CDS) with the corresponding genomic sequences. For phylogenetic tree construction, TIFY protein sequences from watermelon, tomato, rice, and Arabidopsis were aligned by Clustal Omega with default parameters. Then, the alignments of protein sequences were used to construct the phylogenetic tree by MEGA 7.0 using the Neighbor-Joining (NJ) method, with parameters of 1,000 bootstrap replicates and pairwise deletion.

2.3. Chromosomal Location and Duplication Analysis. The genetic positions of watermelon TIFY genes on chromosomes were obtained from the watermelon genome database, and the MapChart software was used to present the chromosomal positions and relative distance of CITIFY genes on the basis of their ascending order of physical position (bp). Gene duplications were conducted using multiple collinear scanning toolkit (MCScanX) software with the default parameters as previously reported [38].

2.4. In Silico Expression Analysis of ClTIFY Genes. The inbred line 97103 strand-specific RNA-seq of both the flesh and rind at four pivotal stages of fruit development (10 days after pollination, $10 \mathrm{DAP} ; 18 \mathrm{DAP} ; 26 \mathrm{DAP}$; and $34 \mathrm{DAP}$ ) was analyzed [39]. Fragments per kilobase of exon model per million mapped (FPKM) values were $\log _{2}$-transformed and heat maps with hierarchical clustering were plotted using the OmicShare tools (http://www.omicshare.com/tools).

2.5. Plant Materials and Treatments. Watermelon (Citrullus lanatus L. Xinong 8) plants were cultured in a growth room $\left(25^{\circ} \mathrm{C} / 19^{\circ} \mathrm{C}, 12 \mathrm{~h}\right.$ light $/ 12 \mathrm{~h}$ dark, $\left.200 \mathrm{~mol} \mu \mathrm{m}^{-2} \mathrm{~s}^{-1}\right)$. At the four-leaf stage, watermelon seedlings grown in hydroponics with Hoagland's solution were treated with JA and abiotic stresses. For JA treatment, the leaves were sprayed with $100 \mu \mathrm{M}$ methyl jasmonate (MeJA) solution. For abiotic stress treatments, the plants were exposed to $200 \mathrm{mM} \mathrm{NaCl}$ solution for $24 \mathrm{~h}$ (salt stress), or under 20\% PEG-6000 ( $w / v)$ for $24 \mathrm{~h}$ (drought stress) under the same photoperiod and light 
TABLE 1: ClTIFY family genes identified in watermelon.

\begin{tabular}{|c|c|c|c|c|c|c|c|}
\hline Gene name & Gene ID & Map position (bp) & CDS length & Protein length (aa) & MW (kDa) & $\mathrm{pI}$ & TIFY motif \\
\hline ClJAZ1 & Cla009781 & Chr1:32842754:32843948 & 357 & 118 & 13.05 & 10.13 & TIFYNG \\
\hline ClJAZ2 & Cla019575 & Chr3:7148182:7148833 & 438 & 145 & 16.37 & 7.64 & TIFYNG \\
\hline ClJAZ3 & Cla011143 & Chr3:25698497:25699599 & 795 & 239 & 25.35 & 10.29 & TIFYAG \\
\hline ClJAZ4 & Cla018445 & Chr4:22124533:22128318 & 720 & 365 & 38.73 & 9.58 & TIFYAG \\
\hline ClTIFY1 & Cla020951 & Chr5:25205424:25208778 & 1203 & 400 & 42.65 & 9.18 & TIFYGG \\
\hline ClJAZ5 & Cla001487 & Chr6:1759629:1760654 & 510 & 169 & 18.51 & 10.56 & TIFYNG \\
\hline ClPPD1 & Cla007531 & Chr6:7067544:7071737 & 1023 & 340 & 37.36 & 7.84 & TIFYCG \\
\hline ClJAZ6 & Cla003391 & Chr7:8397563:8400326 & 1149 & 382 & 39.65 & 9.05 & TIFYGG \\
\hline ClJAZ7 & Cla012536 & Chr7:23895841:23896660 & 639 & 212 & 23.22 & 9.43 & TIFYDG \\
\hline ClZML1 & Cla022146 & Chr8:20879791:20886385 & 1053 & 350 & 38.90 & 4.73 & TLSFEG \\
\hline ClZML2 & Cla022147 & Chr8:20889642:20899056 & 894 & 297 & 31.98 & 6.76 & TLSFRG \\
\hline ClTIFY2 & $\mathrm{Cla} 022284$ & Chr8:22248851:22252368 & 1353 & 450 & 47.43 & 8.91 & TIFYGG \\
\hline ClJAZ8 & Cla015533 & Chr9:695779:698687 & 900 & 299 & 32.22 & 9.80 & TIFYAG \\
\hline ClZML3 & Cla015488 & Chr9:1150618:1154927 & 1089 & 362 & 40.78 & 8.18 & TLSYQG \\
\hline ClZML4 & $\mathrm{Cla} 012926$ & Chr9:21054263:21058204 & 798 & 265 & 28.47 & 5.45 & TLSFRG \\
\hline
\end{tabular}

conditions. Untreated seedlings were used as the controls. The leaves were sampled at different time points $(0 \mathrm{~h}, 1 \mathrm{~h}$, $3 \mathrm{~h}, 9 \mathrm{~h}$, and $24 \mathrm{~h}$ ) after treatment. For the analysis of the transcripts of TIFY genes in different tissues of watermelon, the roots, stems, mature leaves, stem apexes, and fruits were sampled separately from 8 -week-old watermelon plants. All samples were collected and immediately frozen in liquid nitrogen and then stored in $-80^{\circ} \mathrm{C}$ prior to RNA extraction.

2.6. RNA Extraction and Quantitative Real-Time PCR ( $q R T-P C R)$. Total RNA was extracted using the total RNA Miniprep Kit (Axygen Biosciences, Union City, CA, USA) according to the manufacturer's instructions. Single-stranded cDNAs were synthesized following the manufacturer's instructions (ReverTra Ace qPCR RT Kit, Toyobo, Japan). The gene-specific primers are shown in Supplementary Table S1. qRT-PCR was performed with the iCycler $\mathrm{iQ}^{\mathrm{TM}}$ Real-Time PCR Detection System (Bio-Rad, Hercules, CA, USA). The PCR was run at $95^{\circ} \mathrm{C}$ for $3 \mathrm{~min}$, followed by 40 cycles of $30 \mathrm{~s}$ at $95^{\circ} \mathrm{C}, 30 \mathrm{~s}$ at $58^{\circ} \mathrm{C}$, and $1 \mathrm{~min}$ at $72^{\circ} \mathrm{C}$. The watermelon Actin gene (Cla007792) was used as an internal control. Each treatment was performed with three independent biological replicates and three technical replicates. Relative expression levels were calculated as described previously [40].

2.7. Statistical Analysis. All statistical analyses were conducted using the SPSS18 statistical package (Chicago, IL, USA). The data were subjected to one-way analysis of variance (ANOVA), and means were compared by Tukey's multiple comparisons test. $P<0.05$ was accepted as significant, and the differences between treatment means are indicated by different letters.

\section{Results}

3.1. Identification and Characterization of TIFY Family Members in Watermelon. A total of 15 ClTIFY family genes were identified in the watermelon genome, which were further examined using SMART and PFAM to confirm that their coding protein sequences contain the TIFY, Jas, ZIM, and PPD domains. The results revealed that these 15 CITIFY genes consisted of eight ClJAZs, four ClZMLs, two ClTIFYs, and one CIPPD. These genes were named according to their chromosomal positions and the domains that they contained as described in other plant species $[6,34,35]$. The amino acid sequences of TIFY family members varied from 118 aa (ClJAZ1) to $450 \mathrm{aa}$ (ClTIFY2) in length, from $13.05 \mathrm{kDa}$ (ClJAZ1) to $47.43 \mathrm{kDa}$ (ClTIFY2) in theoretical MW, and from 4.73 (ClZML1) to 10.56 (ClJAZ5) in pI values (Table 1).

3.2. Phylogenetic Characterization of Watermelon TIFY Gene Family. To study the evolutionary relationship of the watermelon TIFY gene family, a phylogenetic tree was created from the 72 TIFY protein sequences, including 18 in Arabidopsis, 20 in rice, 19 in tomato, and 15 in watermelon. As a result, these TIFY proteins could be classified into four subfamilies: JAZ, ZML, TIFY, and PPD (Figure 1), among which the JAZ subfamily was the largest and could be further divided into five groups (JAZ I-V). It is noteworthy that the JAZ group I was close to the ZML subfamily, which was composed of ZIM and ZML proteins including four in watermelon, four in rice, three in Arabidopsis, and three in tomato, respectively. Three JAZ groups (I, III, and V) comprised JAZ proteins from Arabidopsis, rice, tomato, and watermelon; JAZ group IV included JAZ proteins from Arabidopsis, rice, and tomato; whereas JAZ group II only contained OsJAZs (Figure 1). The PPD proteins constituted a unique clade, including two members from Arabidopsis (AtPPD1 and AtPPD2), two from tomato (SIPPD1 and SIPPD2), and only one from watermelon (CIPPD1). The TIFY subfamily consisted of two TIFY proteins from watermelon, and one TIFY protein from each of Arabidopsis, rice, and tomato. It should be noted that the watermelon CITIFY proteins were more closely related to the TIFY proteins from Arabidopsis and tomato than to those from rice (Figure 1). 


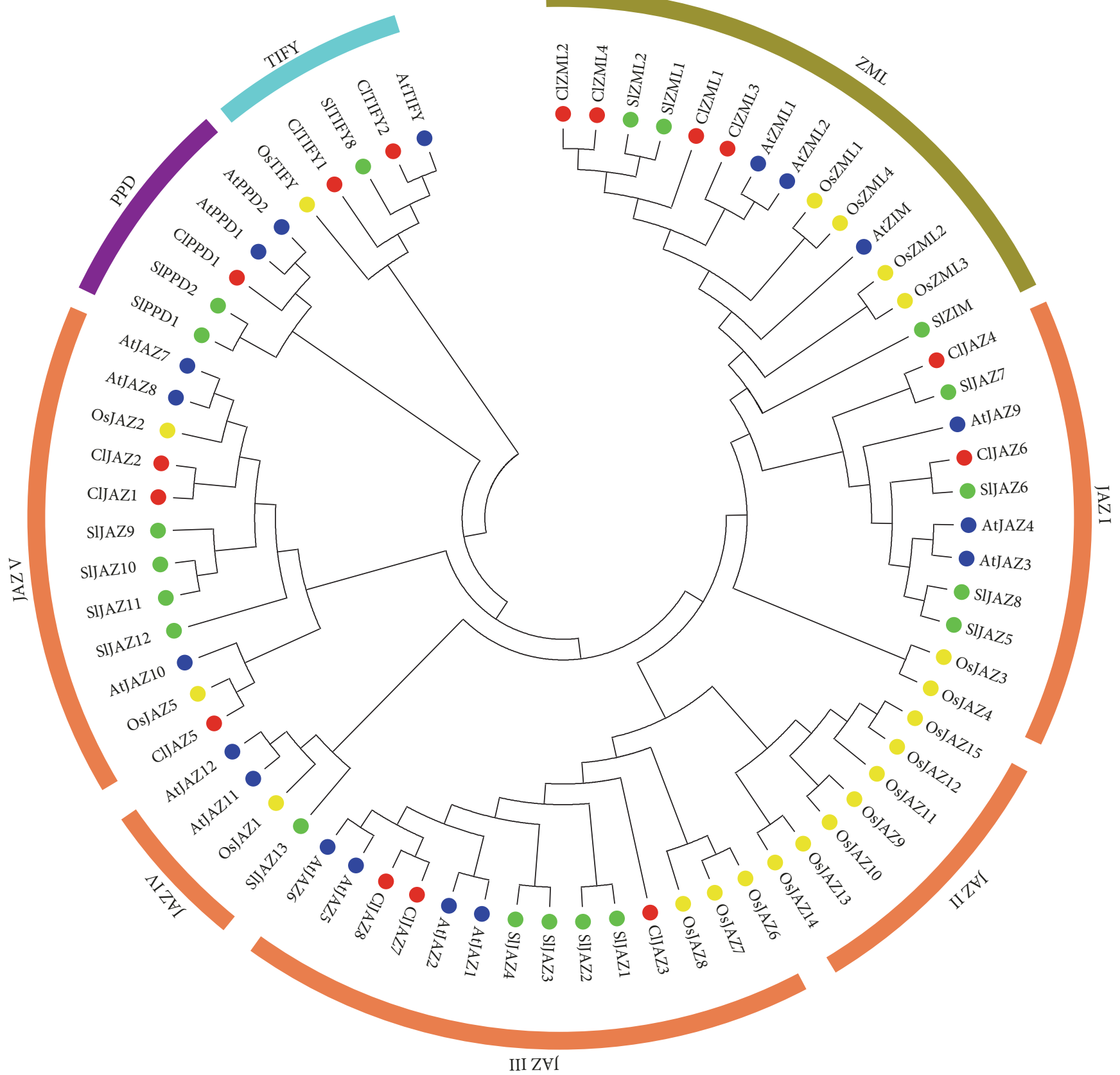

FIgURE 1: Phylogenetic relationships of TIFY family proteins of watermelon, tomato, rice, and Arabidopsis. The phylogenetic tree was created by MEGA 7.0 using the NJ method with 1000 bootstrap replicates.

3.3. Protein Architecture and Conserved Domain Analysis of TIFYs in Watermelon. The protein sequence analysis showed that all the watermelon TIFY proteins contained the TIFY domain (PF06200), with the conservation at the amino acid level of TIFYXG, except for ClZMLs, whose TIFY domain sequences were TLS(F/Y)XG (Table 1; Figure S1). In addition, thirteen of these 15 watermelon TIFY proteins (except for ClTIFY1 and ClTIFY2) possessed the Jas domain (PF09425). The conserved sequences of ClJAZs and ClZMLs were SLXRF(L/F)(E/Q)KRKXRX ${ }_{5}$ PY and SLXRFR(E/Q)KRKXRX 7 Y, while ClPPD contained a truncated Jas motif lacking the conserved $\mathrm{P}$ and $\mathrm{Y}$ residues (Figure S1). Moreover, the GATA zinc-finger (ZnF_GATA, PF00320) domain was represented by $\mathrm{CX}_{2} \mathrm{CX}_{20} \mathrm{CX}_{2} \mathrm{C}$ in all the four ClZML proteins (Figure S1), which was also described in Arabidopsis [23] and pigeon pea [31].

To further explore the diversity of the conserved domains of watermelon TIFY proteins, the MEME online tool was employed, and 10 conserved motifs were identified and named as motifs $1-10$. Among them, motifs 1 and 5 or motifs 1 and 10 composed the TIFY domain, and motif 2 was annotated as the Jas domain (Figure 2). Nearly all watermelon 


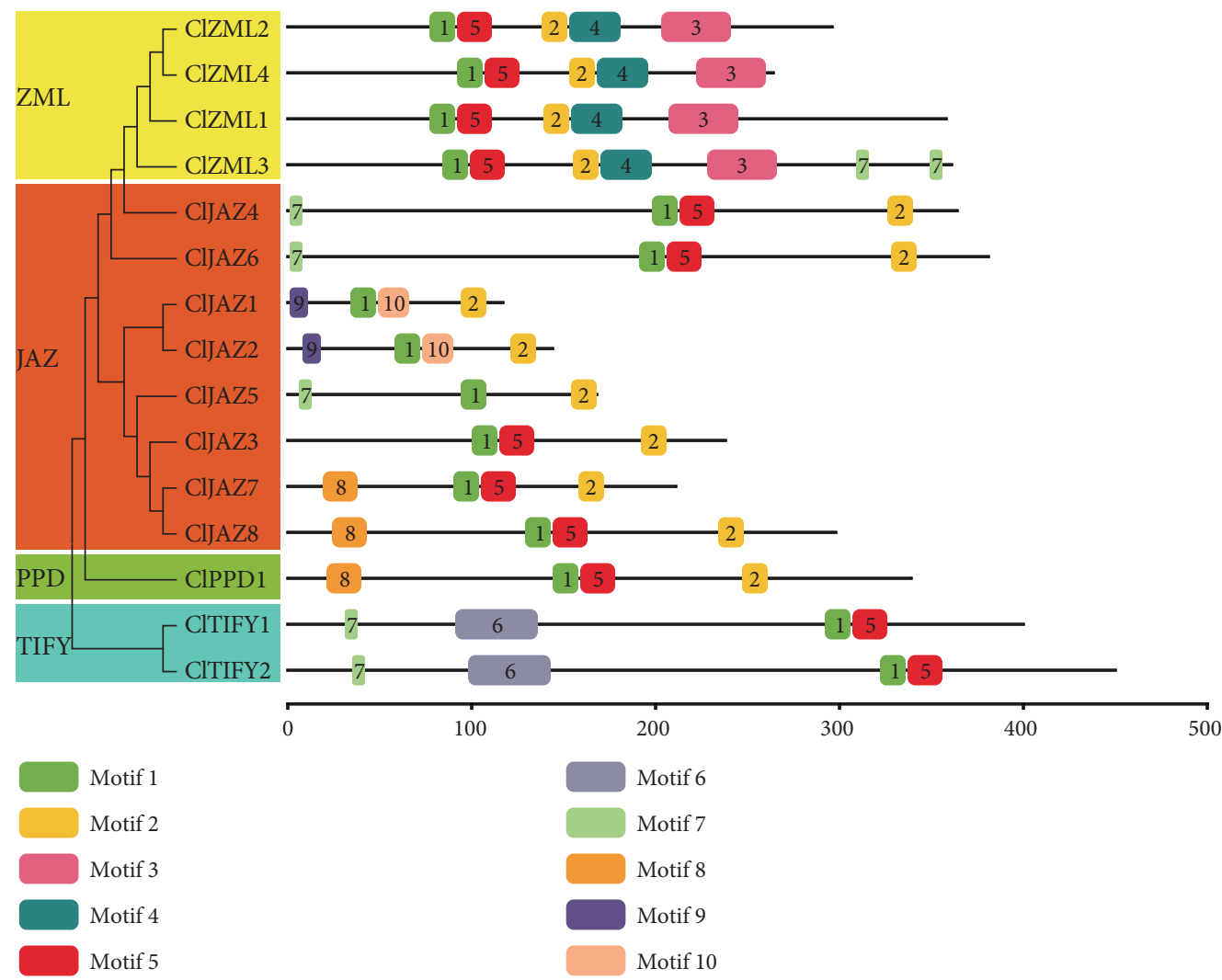

Figure 2: Conserved domains of CITIFYs based on the evolutionary relationship. Different motifs are presented by different colors, and the lengths of the motifs in each protein are shown proportionally.

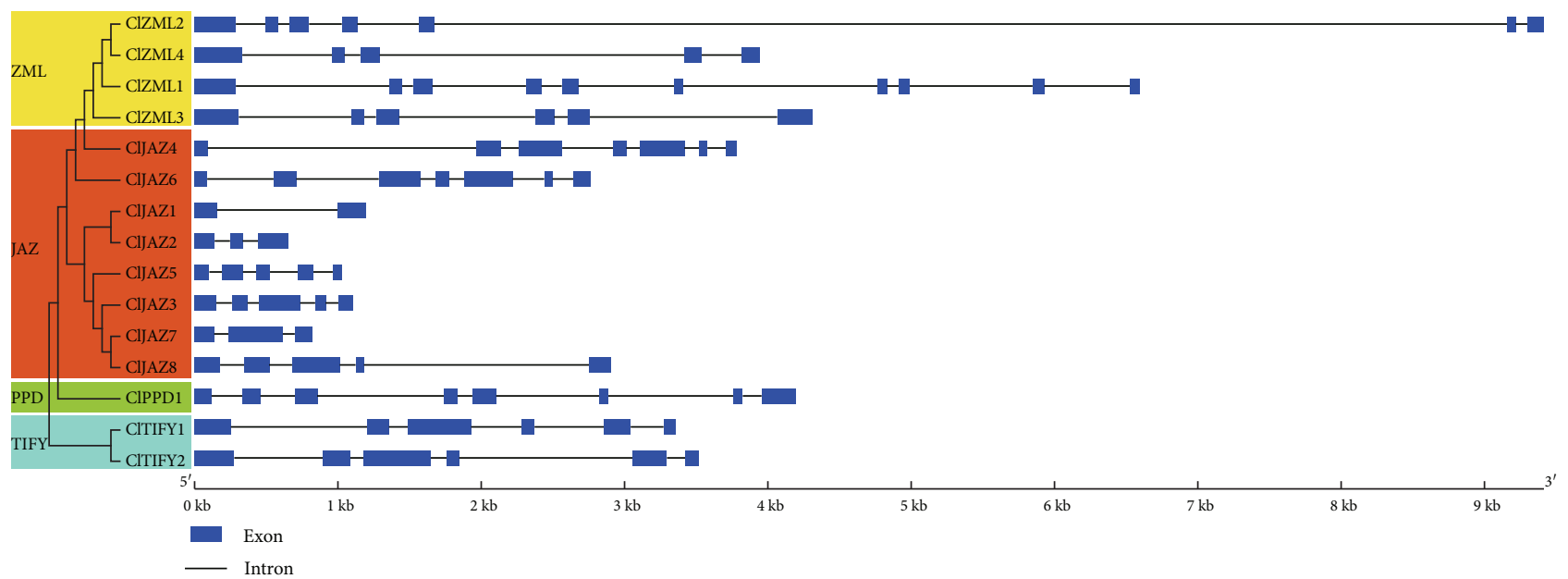

FIGURE 3: Exon-intron structures of CITIFY genes based on the evolutionary relationship. Blue boxes and black lines represent the exons and introns, respectively.

TIFY proteins contained motifs 1 and 2, with the exception of CITIFY1 and CITIFY2, both of which lacked motif 2 but harbored an additional motif 6 . In addition, motif 3 was annotated as the ZnF_GATA domain, motifs 2 and 4 made up the CCT domain, and both domains were only present in the ClZML proteins. In addition, both ClJAZ1 and ClJAZ2 contained motif 9 at their N-terminus, which was associated with a classical EAR motif (LXLXL) (Figure 2; Table S2).
Moreover, some watermelon TIFY proteins had unique motifs. For example, motif 8 was observed in ClJAZ7, ClJAZ8, and CIPPD1 but was absent in other watermelon TIFY proteins (Figure 2).

3.4. Gene Structure Analysis of CITIFY Genes. To understand the structural components of the CITIFY genes, the gene structures of the CITIFY genes were analyzed by alignment 


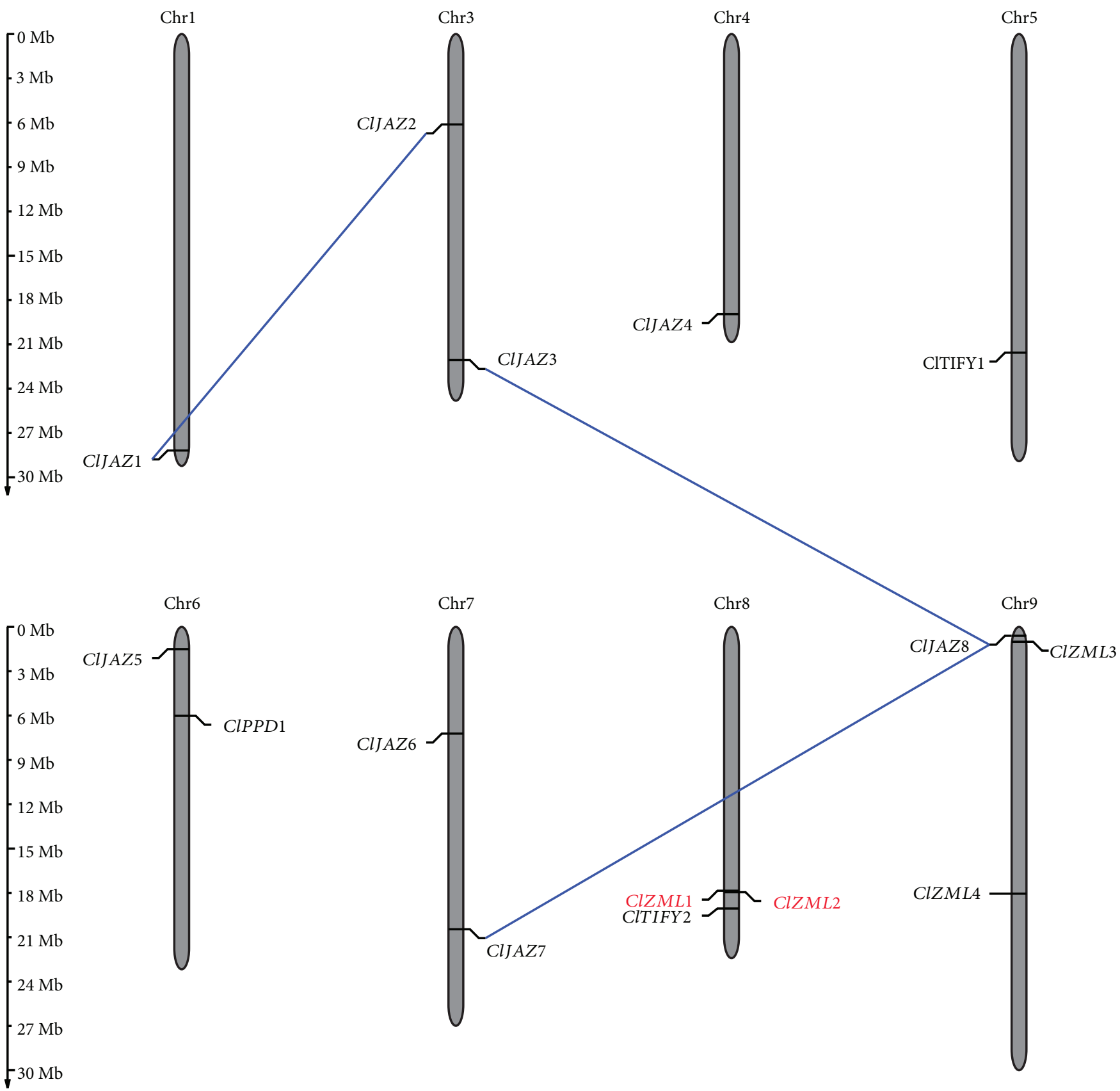

FIgure 4: Chromosomal distribution of ClTIFY genes. The ClTIFY genes located in duplicated chromosomal segments are connected by broken lines. Only those chromosomes containing ClTIFY genes are represented. The tandemly duplicated genes are marked in red.

with the CDS sequences and corresponding genomic DNA sequences. The results revealed that the CITIFY genes contained 1-9 introns, and the number of introns in each subfamily varied. For example, in the JAZ subfamily, ClJAZ4 and ClJAZ6 had six introns; ClJAZ3, ClJAZ5, and ClJAZ8 harbored four introns; ClJAZ2 and ClJAZ7 contained two introns; while ClJAZ1 possessed only one intron (Figure 3). It can be observed that all $Z M L$ subfamily genes have longer genomic sequences than the TIFY genes of other subfamilies, and the intron number of ClZMLs was 4-9. However, some members in the same subfamily shared similar intron numbers but with different intron lengths, such as ClJAZ4 and
ClJAZ6, ClJAZ3 and ClJAZ5, and ClTIFY1 and ClTIFY2 (Figure 3).

3.5. Chromosome Distribution and Duplication Analysis of ClTIFY Genes. The 15 ClTIFY genes were distributed among eight chromosomes, with chromosomes 8 and 9 containing the most genes (three genes for each), followed by chromosomes 3, 6, and 7 (two genes for each), while chromosomes 1,4 , and 5 contained the fewest ClTIFY genes (one gene for each) (Figure 4). The segmental and tandem duplication events among the ClTIFY genes were further determined. As a result, three paralogous gene pairs (ClJAZ1/ClJAZ2, 

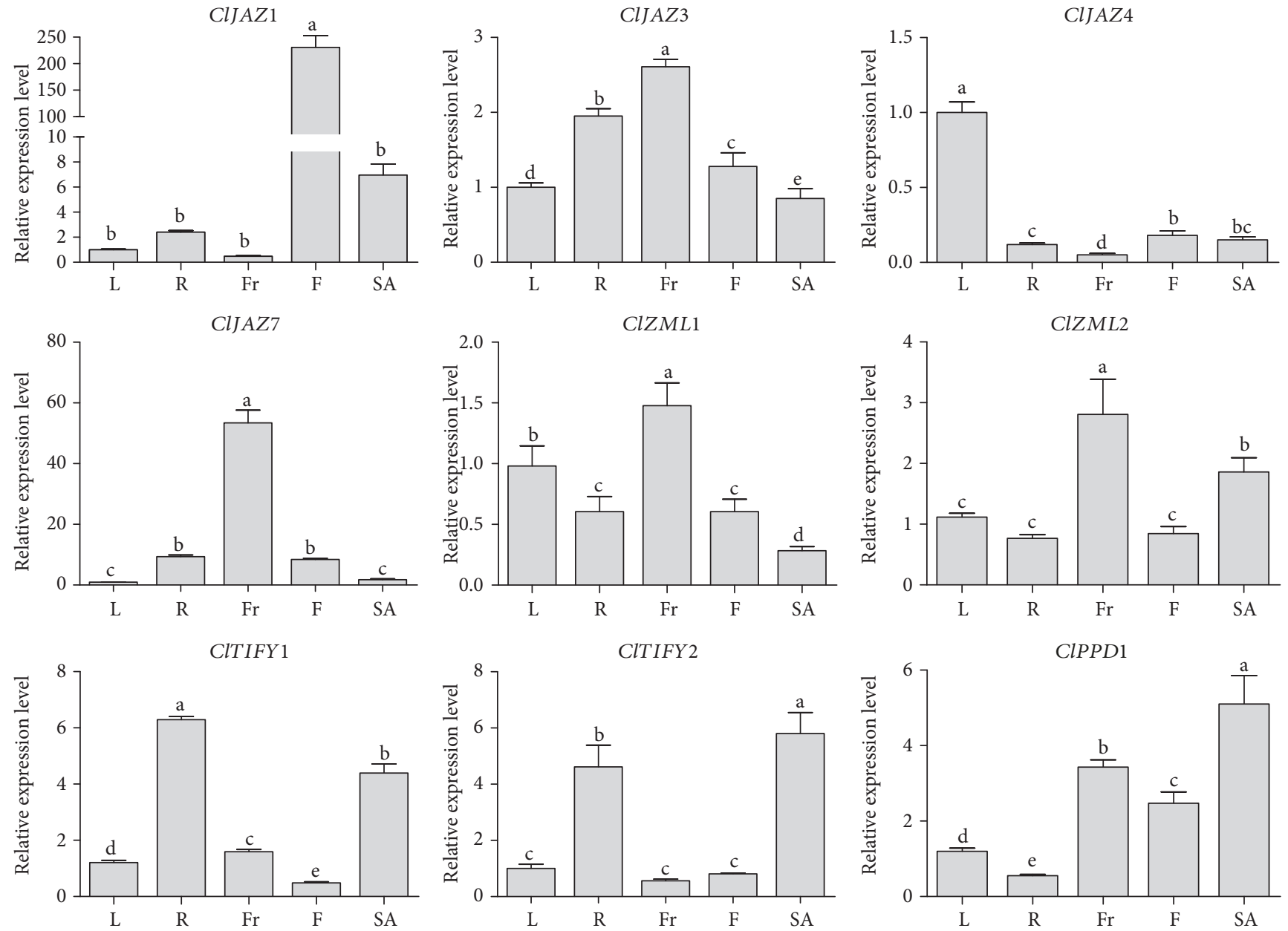

FIGURE 5: qRT-PCR analysis of the expression patterns of nine ClTIFY genes in different tissues of watermelon. L: leaves; R: roots; Fr: fruits; F: flowers; SA: stem apexes. The vertical axis represents relative expression levels and error bars indicate standard deviation (SD) of three independent experiments. Columns with different letters are significantly different $(P<0.05)$.

ClJAZ3/ClJAZ8, and ClJAZ7/ClJAZ8) were found to be related to segmental duplication events, while only one tandem duplication event was identified on chromosome 8 (ClZML1/ClZML2).

3.6. Tissue-Specific Expression Profiles of the ClTIFY Genes. To gain insights into the expression profiles of the ClTIFY genes in various tissues, qRT-PCR analysis was carried out to examine the expression of nine selected ClTIFY genes in leaves, roots, fruits, flowers, and stem apexes. The qRTPCR data revealed the preferential expression of these ClTIFY genes (Figure 5). Among them, ClJAZ1, ClJAZ4, and ClJAZ7 were highly and preferentially expressed in flowers, leaves, and fruits, respectively. In addition, ClJAZ3, ClZML1, and ClZML2 had higher expression in fruits than in other tissues. Interestingly, two TIFY subfamily members, ClTIFY1 and ClTIFY2, were highly expressed in roots and stem apexes (Figure 5). Additionally, ClPPD1 expression was also high in stem apexes, but relatively lower in fruits, flowers, and other tissues. The results showed that the CITIFY genes had overlapping but spatially varying expres- sion, indicating that they may play important roles in specific tissues.

3.7. Characterization of the Expression of ClTIFYs during Watermelon Fruit Development. The expression profiles of ClTIFYs during fruit development in watermelon were analyzed according to the transcriptome data from a previous study [39]. The results showed that four CITIFY genes were differentially expressed during watermelon flesh and rind development (Figure 6). During flesh development, ClZML1 showed low expression all the time, while ClJAZ4 showed an observable accumulation of transcripts. During the development of rind, ClJAZ4 and ClJAZ8 exhibited specifically higher expression at some time points, while ClJAZ5 showed lower transcripts at all stages of rind development (Figure 6). These findings indicated that these genes might play a role in the fruit development of watermelon.

3.8. Expression Profiles of ClTIFY Genes in response to Salt, Drought, and JA Treatments. To gain more insights into the roles of CITIFY genes in response to hormone and various stresses, the expression of nine selected ClTIFY genes was 

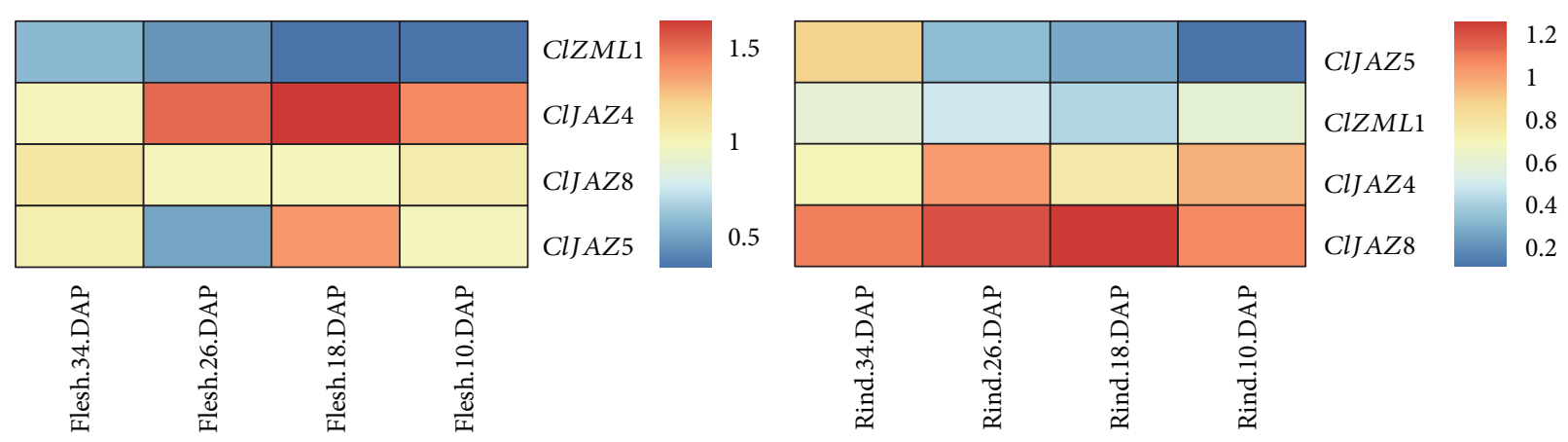

(a)

(b)

Figure 6: Cluster analysis of the expression of four CITIFY genes during the development of flesh and rind at different stages. The expression of TIFY genes was determined from the RNA-seq data. Scale bars on the right of each heat map represent $\log _{2}$ FPKM-transformed (treatment/control) values. Blue, yellow, and red colors indicate low, regular, and high signal intensity, respectively.

ClJAZ1
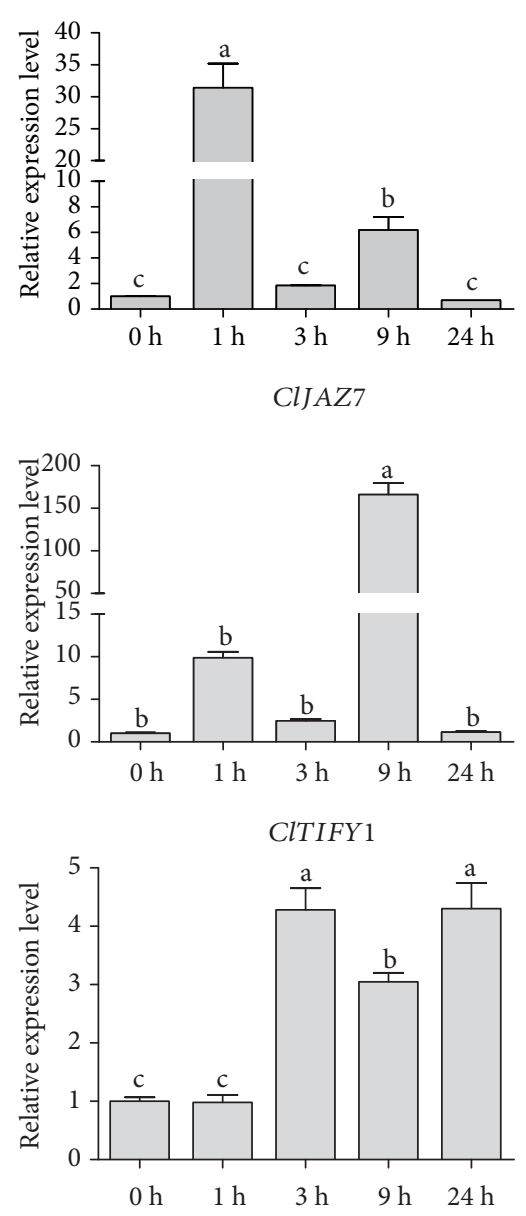

ClJAZ3
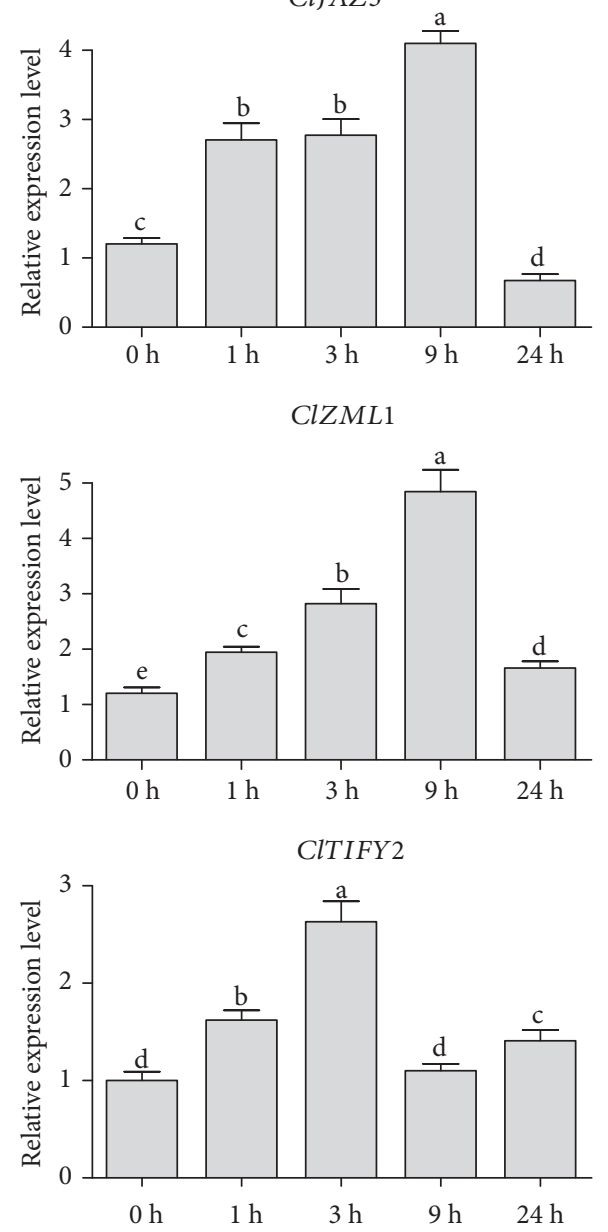

ClJAZ4
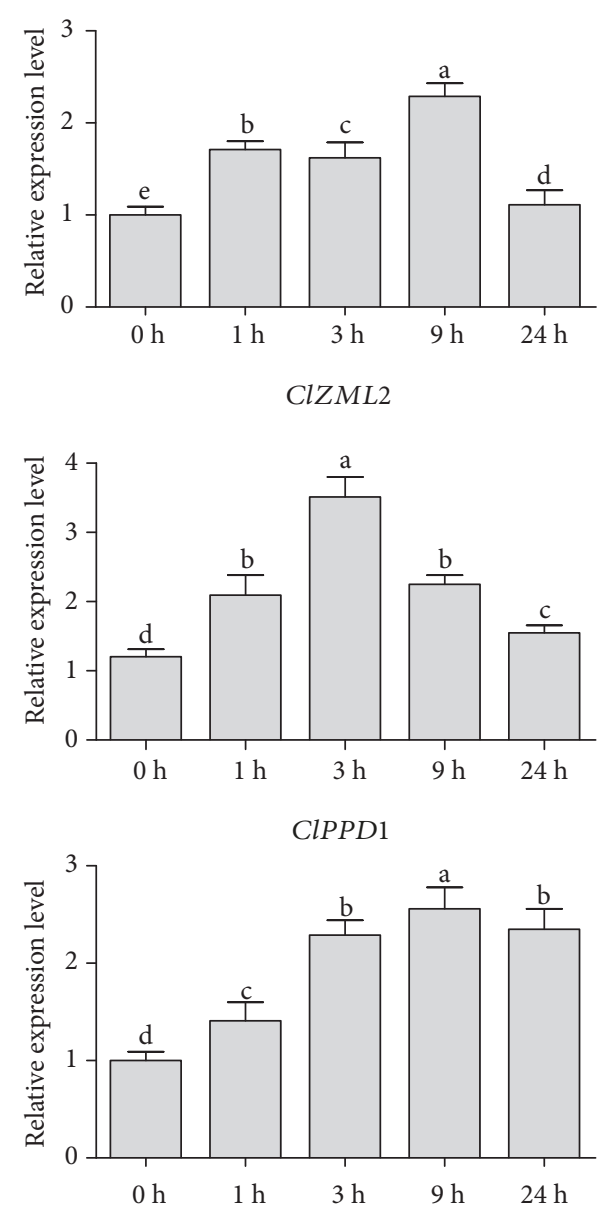

Figure 7: Differential expression detected for nine ClTIFY genes in response to drought treatment at $0 \mathrm{~h}, 1 \mathrm{~h}, 3 \mathrm{~h}, 9 \mathrm{~h}$, and $24 \mathrm{~h}$. The relative expression levels of the selected ClTIFY genes were examined by qRT-PCR. Error bars denote the SD calculated from three independent experiments. Columns with different letters are significantly different $(P<0.05)$.

analyzed by qRT-PCR under various stress treatments including salt, drought, and JA. The results suggested that most of the genes exhibited obvious variations in expression under these treatments. Under drought stress, the expression of all detected CITIFY genes was significantly upregulated
(Figure 7). Among them, ClJAZ7 and ClJAZ1 showed 166and 31-fold increases at specific time points after drought treatment, respectively, while the other seven CITIFY genes exhibited 2- to 5 -fold increases in expression compared with the control (Figure 7). 

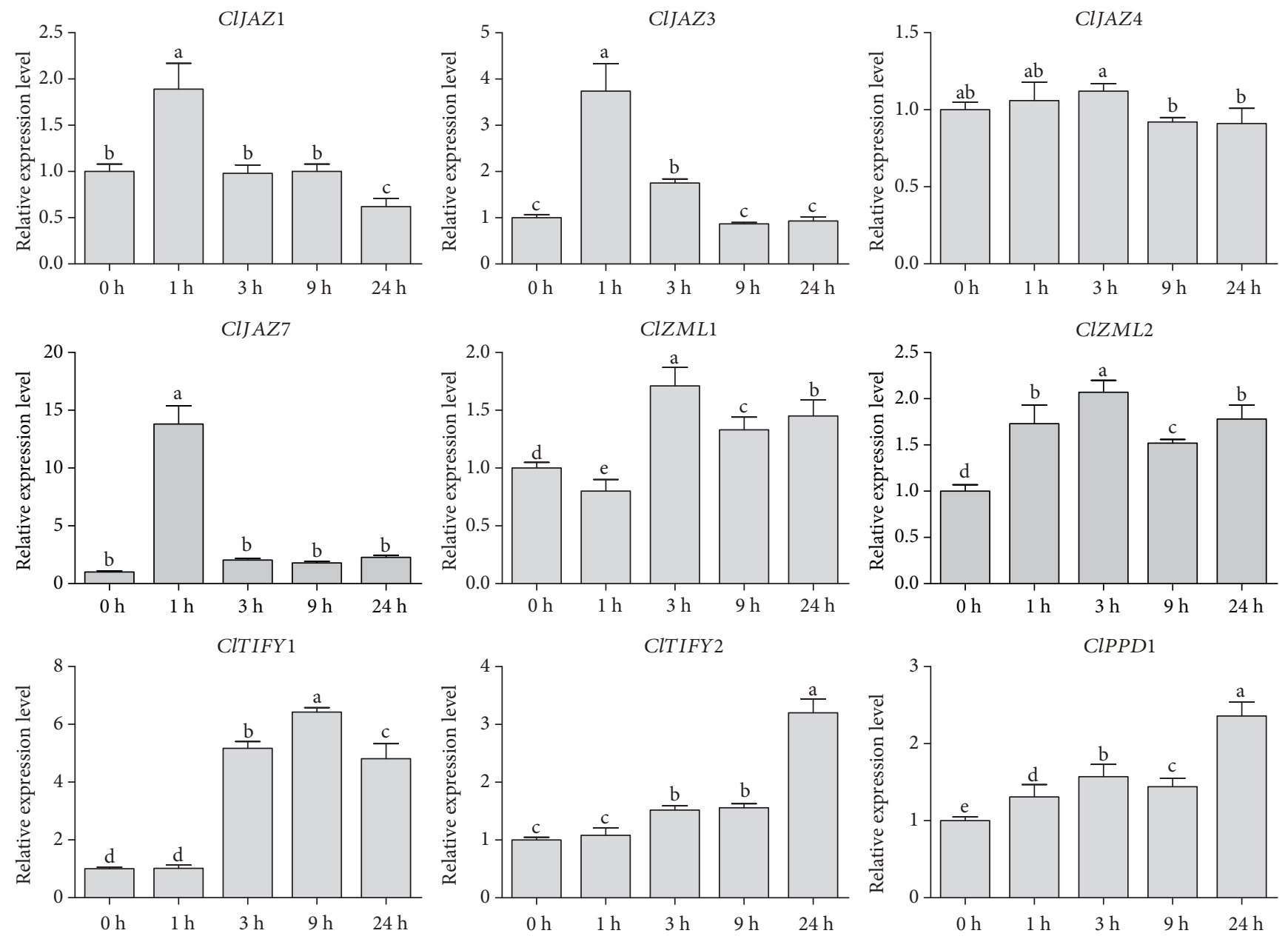

Figure 8: Differential expression detected for nine ClTIFY genes in response to salt treatment at $0 \mathrm{~h}, 1 \mathrm{~h}, 3 \mathrm{~h}, 9 \mathrm{~h}$, and $24 \mathrm{~h}$. Bars represent mean value $\pm \mathrm{SD}$ of three independent experiments. Columns with different letters are significantly different $(P<0.05)$.

It was observed that nearly all the detected CITIFY genes were highly induced by salt treatment, with the exception of ClJAZ4, which seemed to be insensitive to salt treatment (Figure 8). Notably, the transcripts of ClJAZ1, ClJAZ3, and ClJAZ7 displayed more dramatic increases at $1 \mathrm{~h}$ than at other time points after treatment (Figure 8), implying that they play vital roles in the response of watermelon to salt stress in earlier periods.

Upon JA treatment, eight out of the nine selected ClTIFY genes exhibited significantly upregulated expression, except for ClPPD1, whose expression was significantly reduced at all time points (Figure 9). Among the upregulated ClTIFY genes, ClJAZ1 and ClJAZ7 were the most significantly induced ones, exhibiting 533- and 77-fold changes, respectively.

\section{Discussion}

In the present study, we systematically identified 15 TIFY family genes from the watermelon genome, including eight ClJAZs, four ClZMLs, two ClTIFYs, and one ClPPD. Since only one $P P D$ gene was present in the watermelon genome, the number of TIFY genes in watermelon was smaller than that in other plant species, whose numbers of TIFY genes range from 18 to 54, such as Arabidopsis (18) [37], rice (20) [2], Brachypodium distachyon (21) [28], pear (21) [35], poplar (24) [6, 34], maize (30) [29], Brassica rapa (36) [5], wheat (49) [16], Gossypium hirsutum (50), and G. barbadense (54) [33]. This difference might be attributed to gene duplication events, including large segmental duplications and small-scale tandem duplications, which have been proven to play a key role in the expansion and function diversification of genes in TIFY gene family [2, 28, 41]. In this study, only three segmental duplications and one tandem duplication were observed (Figure 4), and the number is much smaller than that in other plants, indicating that the identified TIFY genes are indispensable for the growth and development of watermelon.

Phylogenetic analysis showed that watermelon TIFY proteins can be divided into four subfamilies (JAZ, ZML, TIFY, and PPD), and the JAZ subfamily can be further classified into five groups (Figure 1). It was observed that some JAZ proteins were clustered in monocot- or eudicot-specific patterns, suggesting that these JAZ proteins might have predated the divergence between monocotyledonous and dicotyledonous plants (Figure 1). Multiple sequence alignments showed 

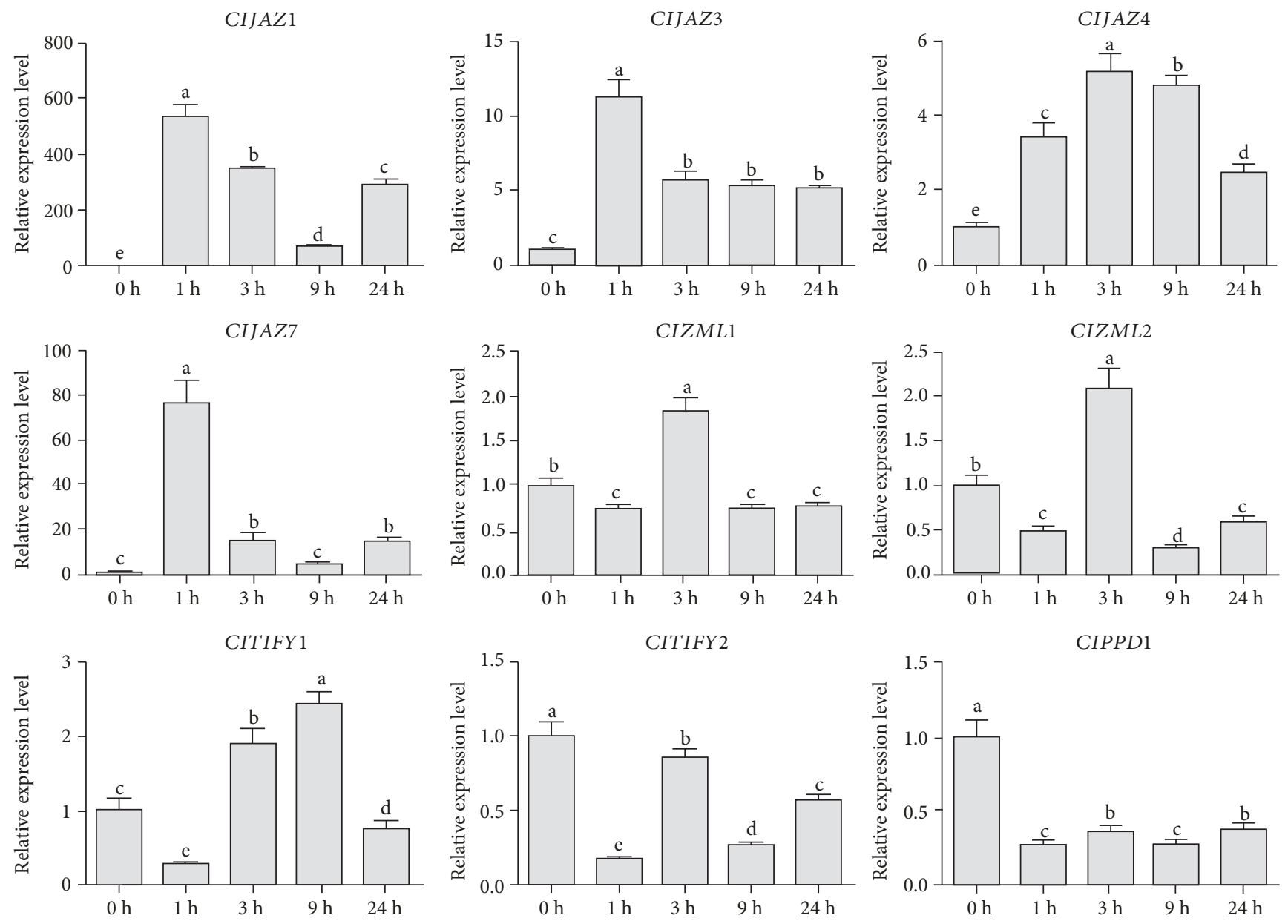

Figure 9: Differential expression detected for nine ClTIFY genes in response to JA treatment at $0 \mathrm{~h}, 1 \mathrm{~h}, 3 \mathrm{~h}, 9 \mathrm{~h}$, and $24 \mathrm{~h}$. Bars represent mean value $\pm \mathrm{SD}$ of three independent experiments. Columns with different letters are significantly different $(P<0.05)$.

that the Jas motif of ClJAZs is strikingly similar to the special consensus sequence of $\mathrm{SLX}_{2} \mathrm{FX}_{2} \mathrm{KRX}_{2} \mathrm{RX}_{5} \mathrm{PY}$ in other plants $[3,5]$, which has been reported to participate in the formation of the JA-Ile-COI1-JAZ complex [42]. However, the sequence of conserved Jas domains from ClZML proteins was $\operatorname{SLXRFR(E/Q)KRKXRX} \mathrm{K}_{7} \mathrm{Y}$, which is slightly different from the characteristic sequence (Figure S1), suggesting that ClJAZs and ClZMLs might play different roles in regulating jasmonate responses in watermelon. We further detected 10 conserved motifs by MEME, and some conserved motifs were unique to specific subfamilies in watermelon TIFY proteins (Figure 2), which may contribute to the function diversification of TIFY proteins. Considering that the exon/intron organization can provide additional clues for understanding the evolutionary relationships among gene families [43], the gene structures of CITIFY genes were determined in this study. Similar to the case in other plants $[1,31], C l Z M L$ genes have longer structures and a larger number of introns than other genes (Figure 3). Besides, ClZML genes exhibited a relatively similar pattern of exon/intron organization but had different intron numbers, indicating that gain or loss of introns occurred during the evolution of ClZML genes, which may lead to the functional divergence $[43,44]$.
Moreover, the JAZ subfamily genes exhibited the highest variability in exon/intron organization, while ClJAZ4 and ClJAZ6 had the same number of introns and similar lengths of exons (Figure 3), suggesting that these two genes are highly conserved during evolution and may have similar functions.

The signal molecule JA plays vital roles in plant growth, development, and responses to environmental stresses. Previous studies have revealed that the TIFY genes play vital roles in various biological processes of plants, such as petiole and hypocotyl elongation [4], lamina size and curvature $[9,11]$, flower development $[13,25,45]$, and seed germination [46]. TIFYs may regulate plant development through the JA signaling pathway. For example, some JAZ proteins can interact with the WD-Repeat/bHLH/MYB complexes to repress JA-mediated trichome initiation and anthocyanin accumulation in Arabidopsis [47]. In Arabidopsis, JAZ4 and JAZ8 competitively interact with WRKY57 to mediate JA-induced leaf senescence [48]. In this study, all the selected CITIFY genes were regulated by JA and exhibited preferential expression in specific tissues (Figures 5 and 9), suggesting that these genes play specific roles in regulating the normal development of plants probably through the JA signaling pathway. It is noteworthy that a majority of ClTIFY 
genes were highly expressed in flowers and fruits (Figure 5), and four ClTIFY genes might play crucial roles in watermelon fruit development (Figure 6), suggesting that ClTIFY genes may function in the development of the flower and fruit of watermelon. It should be noted that abiotic stress can increase the JA content, and TIFY genes were also shown to play vital roles in response to abiotic stresses through the JA signaling pathway. For example, overexpression of apple MdJAZ2 in Arabidopsis decreased JA sensitivity and increased the tolerance to salt and drought stresses during seedling development [49]. Moss PnJAZ1 acts as a repressor to mediate the crosstalk between JA and ABA signaling pathways, and thus increases tolerance to salt stress [21]. In this study, the vast majority of the detected ClTIFYs exhibited differential accumulations under salt and drought stresses. Similar results were also reported in other plants, such as apple [41] and B. rapa [5]. In addition, nearly all the detected ClTIFYs were upregulated by JA treatment, with the exception of ClPPD1, whose expression was downregulated (Figure 9). It is worth noting that ClJAZ1 and ClJAZ7 showed the most remarkable increases in expression under JA and drought treatments (Figures 7 and 9), indicating that they may play essential roles in regulating the response to drought stress by the JA-mediated signaling pathway. ClJAZ1 was clustered together with AtJAZ7 in the JAZ V group (Figure 1), and overexpression of $A t J A Z 7$ was found to confer drought tolerance in Arabidopsis [50]. Under salt treatment, ClJAZ7 exhibited the highest expression among the detected ClTIFYs (Figure 8), demonstrating that it may play a major role in salt stress response. Similarly, overexpression of OsTIFY11a/Os$J A Z 9$ in rice resulted in significantly enhanced tolerance to salt and dehydration stresses, and suppression of OsJAZ9 resulted in reduced salt tolerance through the regulation of JA signaling $[2,15]$. Further studies of these CITIFY genes are needed to unravel their regulatory roles in the development of watermelon and in abiotic stress response through the JA signaling pathway.

\section{Conclusions}

In this study, a total of 15 TIFY genes were identified in the watermelon genome, including eight ClJAZs, four ClZMLs, two ClTIFYs, and one ClPPD. The analysis of qRT-PCR and RNA-seq data revealed that some TIFY genes play organ-specific roles. Expression patterns of the nine selected TIFY genes in response to JA and abiotic stress indicated that they may be involved in abiotic stress response by the JA signaling pathway. Our findings lay a foundation for a further functional characterization of the TIFY family genes in watermelon and a clarification of how TIFY genes can be utilized for the improvement of watermelon via biotechnological strategies.

\section{Data Availability}

The original data of the TIFY family genes are available from the watermelon genome database (http://cucurbitgenomics .org/organism/1).

\section{Conflicts of Interest}

The authors declare that they have no conflict of interest.

\section{Authors' Contributions}

Youxin Yang and Yong Zhou conceived and designed the experiments. Youxin Yang, Rongrong Chen, and Yong Zhou performed the experiments. Youxin Yang, Golam Jalal Ahammed, Chunpeng Wan, Haoju Liu, Rongrong Chen, and Yong Zhou analyzed data. Youxin Yang, Rongrong Chen, and Yong Zhou wrote the manuscript. All authors read and approved the manuscript.

\section{Acknowledgments}

This work was jointly supported by the National Natural Science Foundation of China (31560572), the Natural Science Foundation of Jiangxi Province of China (20171BAB214030), and the Natural Science Foundation of Jiangxi Educational Committee (GJJ160393 and GJJ180172).

\section{Supplementary Materials}

Supplementary 1. Supplementary Fig. S1 Alignment of the amino acid sequences of watermelon TIFY proteins.

Supplementary 2. Supplementary Table S1 Primer sequences used in qRT-PCR.

Supplementary 3. Supplementary Table S2 Sequences and lengths of CITIFY motifs.

\section{References}

[1] Y. Bai, Y. Meng, D. Huang, Y. Qi, and M. Chen, "Origin and evolutionary analysis of the plant-specific TIFY transcription factor family," Genomics, vol. 98, no. 2, pp. 128-136, 2011.

[2] H. Ye, H. Du, N. Tang, X. Li, and L. Xiong, "Identification and expression profiling analysis of TIFY family genes involved in stress and phytohormone responses in rice," Plant Molecular Biology, vol. 71, no. 3, pp. 291-305, 2009.

[3] P. E. Staswick, "JAZing up jasmonate signaling," Trends in Plant Science, vol. 13, no. 2, pp. 66-71, 2008.

[4] M. Shikata, Y. Matsuda, K. Ando et al., "Characterization of Arabidopsis ZIM, a member of a novel plant-specific GATA factor gene family," Journal of Experimental Botany, vol. 55, no. 397, pp. 631-639, 2004.

[5] G. Saha, J.-I. Park, M. A. Kayum, and I.-S. Nou, "A GenomeWide Analysis Reveals Stress and Hormone Responsive Patterns of TIFY Family Genes in Brassica rapa," Frontiers in Plant Science, vol. 7, p. 936, 2016.

[6] Y. Wang, F. Pan, D. Chen, W. Chu, H. Liu, and Y. Xiang, "Genome-wide identification and analysis of the Populus trichocarpa TIFY gene family," Plant Physiology and Biochemistry, vol. 115, pp. 360-371, 2017.

[7] H. S. Chung, Y. Niu, J. Browse, and G. A. Howe, "Top hits in contemporary JAZ: an update on jasmonate signaling," Phytochemistry, vol. 70, no. 13-14, pp. 1547-1559, 2009.

[8] H. Sun, L. Chen, J. Li et al., “The JASMONATE ZIM-domain gene family mediates JA signaling and stress response in 
cotton," Plant and Cell Physiology, vol. 58, no. 12, pp. 21392154, 2017.

[9] D. W. R. White, "PEAPOD regulates lamina size and curvature in Arabidopsis," Proceedings of the National Academy of Sciences of the United States of America, vol. 103, no. 35, pp. 13238-13243, 2006.

[10] H. S. Chung and G. A. Howe, "A critical role for the TIFY motif in repression of jasmonate signaling by a stabilized splice variant of the JASMONATE ZIM-domain protein JAZ10 in Arabidopsis," Plant Cell, vol. 21, no. 1, pp. 131-145, 2009.

[11] A. Baekelandt, L. Pauwels, Z. Wang et al., "Arabidopsis Leaf Flatness Is Regulated by PPD2 and NINJA through Repression of CYCLIN D3Genes," Plant Physiology, vol. 178, no. 1, pp. 217-232, 2018.

[12] N. Gonzalez, L. Pauwels, A. Baekelandt et al., "A Repressor Protein Complex Regulates Leaf Growth in Arabidopsis," Plant Cell, vol. 27, no. 8, pp. 2273-2287, 2015.

[13] J. Tian, L. Cao, X. Chen et al., "The OsJAZ1 degron modulates jasmonate signaling sensitivity during rice development," Development, vol. 146, no. 4, p. dev173419, 2019.

[14] J. Fu, H. Wu, S. Ma, D. Xiang, R. Liu, and L. Xiong, “OsJAZ1 attenuates drought resistance by regulating JA and ABA signaling in rice," Frontiers in Plant Science, vol. 8, p. 2108, 2017.

[15] H. Wu, H. Ye, R. Yao, T. Zhang, and L. Xiong, "OsJAZ9 acts as a transcriptional regulator in jasmonate signaling and modulates salt stress tolerance in rice," Plant Science, vol. 232, pp. 1-12, 2015.

[16] C. Ebel, A. BenFeki, M. Hanin, R. Solano, and A. Chini, "Characterization of wheat (Triticum aestivum) TIFY family and role of Triticum Durum TdTIFY11a in salt stress tolerance," PLoS One, vol. 13, no. 7, p. e0200566, 2018.

[17] H. Liu, T. Li, Y. Wang et al., "TaZIM-A1 negatively regulates flowering time in common wheat (Triticum aestivum L.)," Journal of Integrative Plant Biology, vol. 61, no. 3, pp. 359376, 2019.

[18] X. Yu, G. Chen, B. Tang, J. Zhang, S. Zhou, and Z. Hu, “The Jasmonate ZIM-domain protein gene SlJAZ2 regulates plant morphology and accelerates flower initiation in Solanum lycopersicum plants," Plant Science, vol. 267, pp. 65-73, 2018.

[19] X. He, L. Zhu, G. M. Wassan et al., "GhJAZ2 attenuates cotton resistance to biotic stresses via the inhibition of the transcriptional activity of GhbHLH171," Molecular Plant Pathology, vol. 19, no. 4, pp. 896-908, 2018.

[20] T. Pei, P. Ma, K. Ding et al., "SmJAZ8 acts as a core repressor regulating JA-induced biosynthesis of salvianolic acids and tanshinones in Salvia miltiorrhiza hairy roots," Journal of Experimental Botany, vol. 69, no. 7, pp. 1663-1678, 2018.

[21] S. Liu, P. Zhang, C. Li, and G. Xia, "The moss jasmonate ZIMdomain protein PnJAZ1 confers salinity tolerance via crosstalk with the abscisic acid signalling pathway," Plant Science, vol. 280, pp. 1-11, 2019.

[22] I. Monte, J. M. Franco-Zorrilla, G. Garcia-Casado et al., “A single JAZ repressor controls the jasmonate pathway in Marchantia polymorpha," Molecular Plant, vol. 12, no. 2, pp. 185-198, 2019.

[23] A. Nishii, M. Takemura, H. Fujita, M. Shikata, A. Yokota, and T. Kohchi, "Characterization of a novel gene encoding a putative single zinc-finger protein, ZIM, expressed during the reproductive phase in Arabidopsis thaliana," Bioscience, Biotechnology, and Biochemistry, vol. 64, no. 7, pp. 1402-1409, 2000.
[24] J. Shaikhali, J. de Dios Barajas-Lopéz, K. Ötvös et al., "The CRYPTOCHROME1-dependent response to excess light is mediated through the transcriptional activators ZINC FINGER PROTEIN EXPRESSED IN INFLORESCENCE MERISTEM LIKE1 and ZML2 in Arabidopsis," Plant Cell, vol. 24, no. 7, pp. 3009-3025, 2012.

[25] Q. Cai, Z. Yuan, M. Chen et al., "Jasmonic acid regulates spikelet development in rice," Nature Communications, vol. 5, no. 1, 2014.

[26] S. Taniguchi, Y. Hosokawa-Shinonaga, D. Tamaoki, S. Yamada, K. Akimitsu, and K. Gomi, "Jasmonate induction of the monoterpene linalool confers resistance to rice bacterial blight and its biosynthesis is regulated by JAZ protein in rice," Plant, Cell \& Environment, vol. 37, no. 2, pp. 451-461, 2014.

[27] P. K. Peethambaran, R. Glenz, S. Honinger et al., "Salt-inducible expression of OsJAZ8 improves resilience against saltstress," BMC Plant Biology, vol. 18, no. 1, p. 311, 2018.

[28] L. Zhang, J. You, and Z. Chan, "Identification and characterization of TIFY family genes in Brachypodium distachyon," Journal of Plant Research, vol. 128, no. 6, pp. 9951005, 2015.

[29] Z. Zhang, X. Li, R. Yu, M. Han, and Z. Wu, "Isolation, structural analysis, and expression characteristics of the maize TIFY gene family," Molecular Genetics and Genomics, vol. 290, no. 5, pp. 1849-1858, 2015.

[30] Z. Huang, S. H. Jin, H. D. Guo et al., "Genome-wide identification and characterization of TIFY family genes in moso bamboo (Phyllostachys edulis) and expression profiling analysis under dehydration and cold stresses," PeerJ, vol. 4, article e2620, 2016.

[31] G. Sirhindi, P. Sharma, P. Arya et al., "Genome-wide characterization and expression profiling of TIFY gene family in pigeonpea (Cajanus cajan (L.) Millsp.) under copper stress," Journal of Plant Biochemistry and Biotechnology, vol. 25, no. 3, pp. 301-310, 2016.

[32] A. Chini, W. Ben-Romdhane, A. Hassairi, and M. A. M. Aboul-Soud, "Identification of TIFY/JAZ family genes in Solanum lycopersicum and their regulation in response to abiotic stresses," PLoS One, vol. 12, no. 6, p. e0177381, 2017.

[33] Q. Sun, G. Wang, X. Zhang et al., "Genome-wide identification of the TIFY gene family in three cultivated Gossypium species and the expression of JAZ genes," Scientific Reports, vol. 7, no. 1, 2017.

[34] W. Xia, H. Yu, P. Cao, J. Luo, and N. Wang, "Identification of TIFY family genes and analysis of their expression profiles in response to phytohormone treatments and Melampsora larici-populina infection in poplar," Frontiers in Plant Science, vol. 8, p. 493, 2017.

[35] Y. Ma, S. Shu, S. Bai, R. Tao, M. Qian, and Y. Teng, "Genomewide survey and analysis of the TIFY gene family and its potential role in anthocyanin synthesis in Chinese sand pear (Pyrus pyrifolia)," Tree Genetics \& Genomes, vol. 14, no. 2, 2018.

[36] Q. Kong, J. Yuan, L. Gao et al., "Identification of suitable reference genes for gene expression normalization in qRT-PCR analysis in watermelon," PLoS One, vol. 9, no. 2, p. e90612, 2014.

[37] B. Vanholme, W. Grunewald, A. Bateman, T. Kohchi, and G. Gheysen, "The tify family previously known as ZIM," Trends in Plant Science, vol. 12, no. 6, pp. 239-244, 2007. 
[38] J. You, Y. Wang, Y. Zhang et al., "Genome-wide identification and expression analyses of genes involved in raffinose accumulation in sesame," Scientific Reports, vol. 8, no. 1, 2018.

[39] S. Guo, J. Zhang, H. Sun et al., "The draft genome of watermelon (Citrullus lanatus) and resequencing of 20 diverse accessions," Nature Genetics, vol. 45, no. 1, pp. 51-58, 2013.

[40] K. J. Livak and T. D. Schmittgen, “Analysis of Relative Gene Expression Data Using Real-Time Quantitative PCR and the $2_{\text {T }}^{-\Delta \Delta C}$ Method," Methods, vol. 25, no. 4, pp. 402-408, 2001.

[41] X. Li, X. Yin, H. Wang et al., "Genome-wide identification and analysis of the apple (Malus $\times$ domestica Borkh.) TIFY gene family," Tree Genetics \& Genomes, vol. 11, no. 1, p. 808, 2015.

[42] L. B. Sheard, X. Tan, H. Mao et al., "Jasmonate perception by inositol-phosphate-potentiated COI1-JAZ co-receptor," Nature, vol. 468, no. 7322, pp. 400-405, 2010.

[43] Y. Zhou, L. Hu, S. Ye, L. Jiang, and S. Liu, "Genome-wide identification and characterization of cysteine-rich polycomb-like protein (CPP) family genes in cucumber (Cucumis sativus) and their roles in stress responses," Biologia, vol. 73, no. 4, pp. 425-435, 2018.

[44] G. Xu, C. Guo, H. Shan, and H. Kong, "Divergence of duplicate genes in exon-intron structure," Proceedings of the National Academy of Sciences of the United States of America, vol. 109, no. 4, pp. 1187-1192, 2012.

[45] S. Song, T. Qi, H. Huang et al., "The Jasmonate-ZIM domain proteins interact with the R2R3-MYB transcription factors MYB21 and MYB24 to affect Jasmonate-regulated stamen development in Arabidopsis," Plant Cell, vol. 23, no. 3, pp. 1000-1013, 2011.

[46] L. Ju, Y. Jing, P. Shi et al., "JAZ proteins modulate seed germination through interaction with ABI5 in bread wheat and Arabidopsis," New Phytologist, vol. 223, no. 1, pp. 246-260, 2019.

[47] T. Qi, S. Song, Q. Ren et al., “The Jasmonate-ZIM-domain proteins interact with the WD-Repeat/bHLH/MYB complexes to regulate Jasmonate-mediated anthocyanin accumulation and trichome initiation in Arabidopsis thaliana," Plant Cell, vol. 23, no. 5, pp. 1795-1814, 2011.

[48] Y. Jiang, G. Liang, S. Yang, and D. Yu, “Arabidopsis WRKY57 functions as a node of convergence for jasmonic acid- and auxin-mediated signaling in jasmonic acid-induced leaf senescence," Plant Cell, vol. 26, no. 1, pp. 230-245, 2014.

[49] X. H. An, Y. J. Hao, E. M. Li, K. Xu, and C. G. Cheng, "Functional identification of apple MdJAZ2 in Arabidopsis with reduced JA-sensitivity and increased stress tolerance," Plant Cell Reports, vol. 36, no. 2, pp. 255-265, 2017.

[50] L. Meng, T. Zhang, S. Geng, P. B. Scott, H. Li, and S. Chen, "Comparative proteomics and metabolomics of JAZ7mediated drought tolerance in Arabidopsis," Journal of Proteomics, vol. 196, pp. 81-91, 2019. 


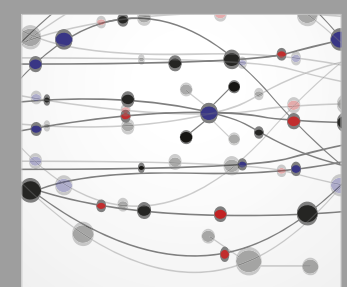

The Scientific World Journal
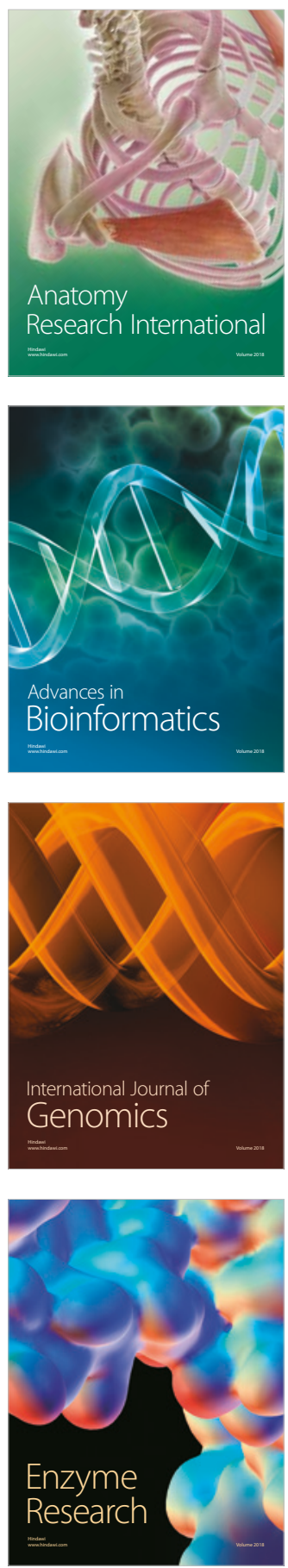
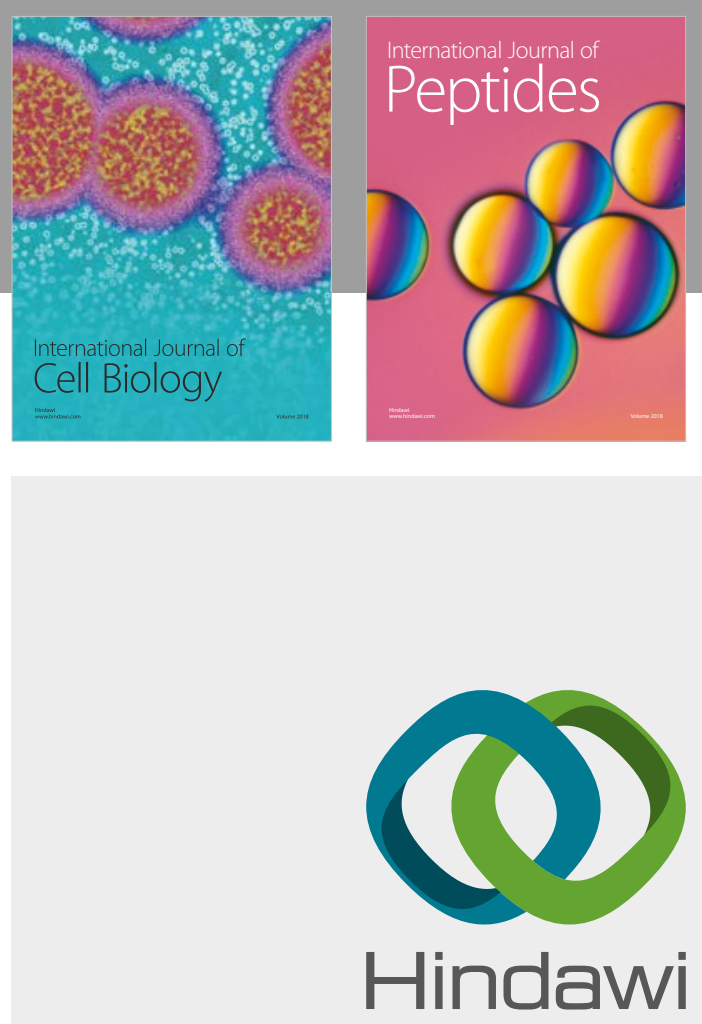

Submit your manuscripts at

www.hindawi.com
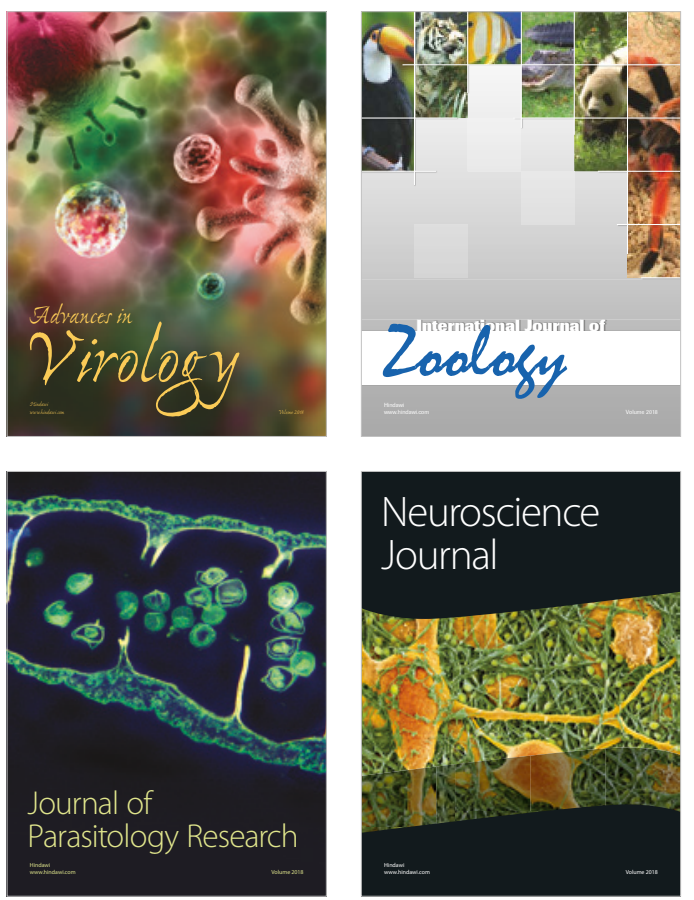
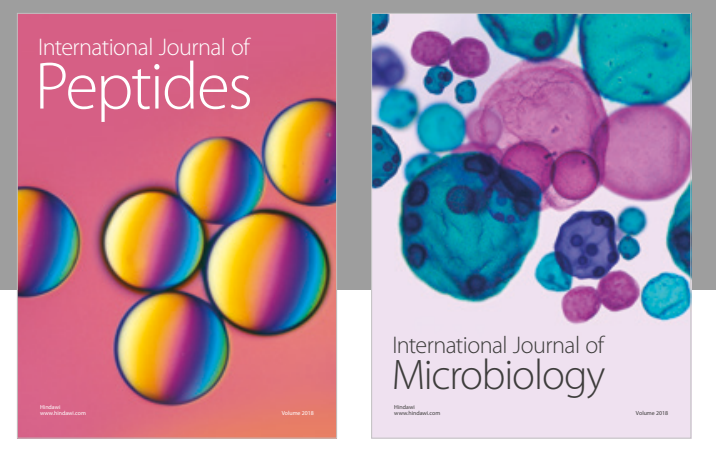

nternational Journal of Microbiology
Journal of
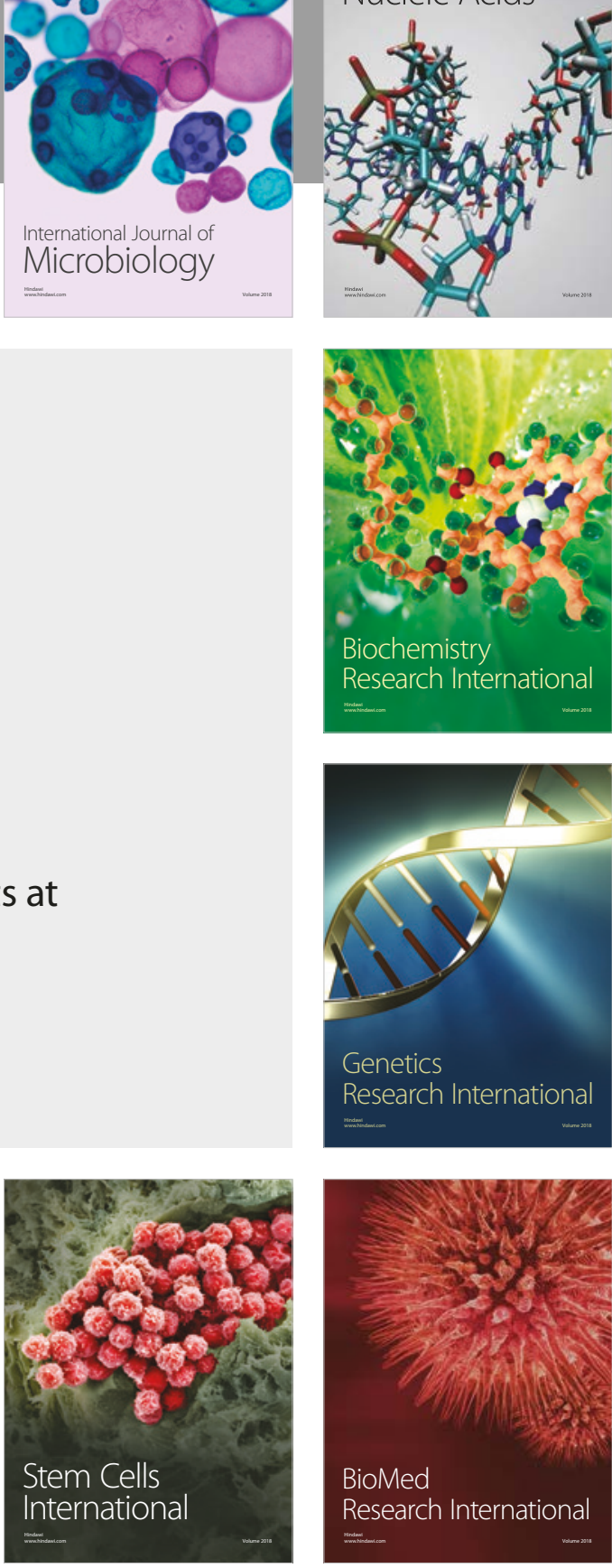
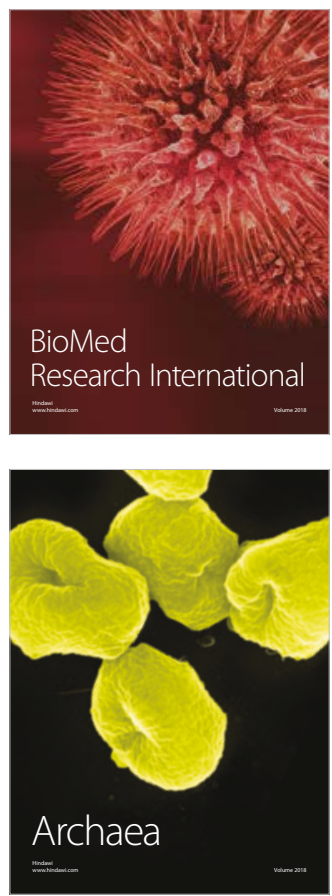